\title{
MODELING UNDERWATER SOUND PROPAGATION FROM AN AIRGUN ARRAY USING THE PARABOLIC EQUATION METHOD
}

\author{
Alexander O. MacGillivray ${ }^{1}$ and N. Ross Chapman ${ }^{2}$ \\ ${ }^{1}$ JASCO Applied Sciences, Victoria, BC, Canada \\ ${ }^{2}$ School of Earth and Ocean Sciences, University of Victoria, Victoria, BC, Canada \\ alex@jasco.com, chapman@uvic.ca
}

\begin{abstract}
This article presents a technique for modeling sound propagation from an airgun array, using the parabolic equation (PE) method, that takes into full account the far-field, angle-dependent radiation pattern of the array. This is achieved by generating a PE starting field for the array by summing together shaded, phase-shifted replicas of the PE self-starter. The array starter has been implemented using the RAM parabolic equation model. A validation comparison is presented of field predictions generated using the array starter against exact normal mode solutions for an array source computed using the ORCA model. Examples of synthetic waveform airgun array calculations performed using the array starter are also provided. The method presented in this article can be used to accurately predict pressure waveforms from an airgun array in the ocean environment provided that the modeler knows (or can compute) far-field source signatures for individual airguns in the array.
\end{abstract}

\section{SOMMAIRE}

Cet article présente une technique permettant de modéliser la propagation du son provenant dun réseau de canons à air, en utilisant la méthode de léquation parabolique (EP), qui prend en compte le patron de directivité du réseau en champ lointain. Cette approche est réalisée en créant un champ initial pour la globalité du réseau défini comme la somme des réplicats de départ (pondérés et déphasés) de chacune des sources du réseau. Ce champ initial de réseau a été mis en uvre en utilisant le modèle déquation parabolique RAM. Les prédictions du champ acoustique générées en utilisant cette technique sont comparées aux solutions exactes des modes normaux pour un réseau de sources calculées avec le modèle ORCA. Des exemples de calculs de forme dondes synthétiques obtenues avec le champ initial de réseau sont également présentés. La méthode décrite dans cet article peut être utilisée pour prédire avec précision les formes donde de pression dun réseau de canons à air dans lenvironnement marin, à condition que le modélisateur connaisse (ou puisse calculer) les signatures en champ lointain de chacun des canons à air du réseau.

\section{INTRODUCTION}

Modern ocean acoustic modeling codes are capable of accurately predicting sound propagation in real ocean environments; however, special treatment is required for modeling directional sources, like airgun arrays, since most available codes solve the wave equation for isotropic (non-directional) sources on a finite range/depth grid. When modeling sound propagation from a strongly directional source, like an airgun array, the modeler must take care to properly couple the directionality function of the source to the pressure field computed by the propagation model. Previous efforts at modeling an airgun array using the parabolic equation (PE) method, by DeRuiter et al. [1], assumed an isotropic source and focused on reproducing the time arrival structure of received pulses while ignoring directionality. Accurate modeling of both the amplitude and frequency structure of airgun array sound emissions requires, however, a rigorous treatment of the frequency-dependent directionality function of the source.

Coupling a directional source to a raytrace code is straightforward: each ray is weighted by the far-field directionality function of the source according to the ray launch angle. Coupling a directional source to a purely harmonic propagation modeling code-i.e., using wavenumber integration, normal modes or parabolic equation method - is not so straightforward. In this case, one can generally simulate a directional source by summing together the fields from an array of discrete isotropic sources located near the origin [2]. The amplitudes and phases of the array elements must be chosen to replicate the far-field directionality pattern of the source under investigation. Even so, airgun arrays (and horizontal arrays in general) present a computational problem because the array elements are not all located at the zero-range of the modeling grid. As shown in the next section, this problem can be overcome in normal mode theory by invoking the far-field approximation for an array source. This, in turn, motivates 
our solution for an array starter for the PE method.

\section{THEORY}

Consider a $N$-element planar array situated at depth $z_{s}$ with its geometric centre at $x=y=0$. In the plane $z=z_{s}$, the array elements are located at the coordinates

$$
\Delta \boldsymbol{r}_{m}=\left(x_{m}, y_{m}\right), \quad m=1,2 \ldots N
$$

Each element of this array has complex amplitude/phase $A_{m}$ at some frequency $f$. We seek an expression for the far-field acoustic pressure from such an array in an arbitrarily stratified, range-independent environment where the acoustic pressure can be computed from the normal mode solution to the Helmholtz equation:

$$
p\left(r, z ; z_{s}\right)=\sqrt{\frac{2 \pi i}{r}} \sum_{n=1}^{\infty} \psi_{n}^{*}\left(z_{s}\right) \psi_{n}(z) k_{n}^{-1 / 2} e^{i k_{n} r},
$$

where $\psi_{n}(z)$ are the normal modes, $k_{n}$ is the horizontal wavenumber of mode $n, z_{s}$ is the source depth, and $r$ and $z$ are the usual cylindrical coordinates.

In the horizontally-stratified, range-independent case, the solution to the wave equation is symmetrical with respect to the azimuth angle, $\theta$. Due to the intrinsic directionality of the array, however, the acoustic field is not generally symmetrical with respect to $\theta$. The total field from this array at location $(\boldsymbol{r}, z)=(r, \theta, z)$ is given by

$$
p_{\Sigma}(\boldsymbol{r}, z)=\sum_{m=1}^{N} A_{m} p\left(\left|\boldsymbol{r}-\Delta r_{m}\right|, z\right) .
$$

Let us define the far-field of the array as the region where $|r| \gg|\Delta r|$. Beyond this range, the separation of the array elements perpendicular to the direction of propagation (i.e., out of the $r / z$ plane) becomes unimportant and we need only consider the position of each array element projected onto the $r / z$ plane:

$$
\Delta r_{m}=\Delta \boldsymbol{r}_{m} \cdot \frac{\boldsymbol{r}}{|\boldsymbol{r}|}=x_{m} \cos \theta+y_{m} \sin \theta
$$

In the far-field of the array, the spreading loss terms for the different array elements are approximately the same (i.e., $\sqrt{r-\Delta r_{m}} \approx \sqrt{r}$. From Equation 1 we obtain the following expression for the total field of the array in terms of the normal modes:

$$
\begin{aligned}
p_{\Sigma}(r, z)= & \sqrt{\frac{2 \pi i}{r}} \sum_{n=1}^{\infty} \psi_{n}^{*}\left(z_{s}\right) \psi_{n}(z) k_{n}^{-1 / 2} e^{i k_{n} r} \\
& \times\left[\sum_{m=1}^{N} A_{m} e^{-i k_{n} \Delta r_{m}}\right]
\end{aligned}
$$

The physical interpretation of Equation 4 is straightforward: in the far-field of the array, the normal modes are weighted by the vertical array directionality (the bracketed term) according to the grazing angle $\phi_{n}=\cos ^{-1}\left(k_{n} c / \omega\right)$.
Equation 4 could be used for computing the field from a horizontal array using normal modes; in particular, it would be useful for computing the initial field for an array using one-way coupled or adiabatic modes. Equation 4 could also be used for constructing a PE starting field from the normal modes (i.e., for generating a modal starter). In a rangeindependent environment, however, there is no computational advantage to Equation 4 since Equation 2 gives the exact answer and is just as easy to compute given the mode functions, $\psi_{n}$ (this fact is exploited to validate the array starter approach in Section 3). Instead, for the general case of rangedependent problems, we seek to rewrite Equation 4 in terms of the parabolic equation solution to the Helmholtz equation and solve the inhomogeneous initial value problem for an array source at $z=z_{s}$. We do so in terms of Collins' PE selfstarter.

Following Collins [3]l, recall that the normal modes in Equation 1 satisfy the eigenvalue equation, which we write using operator notation as follows:

$$
k_{0}^{2}(1+X) \psi_{n}=k_{n}^{2} \psi_{n},
$$

where $k_{0}$ is some reference wavenumber, $\rho$ is the density of the medium, and the depth operator $X$ is defined to be

$$
X=k_{0}^{-2}\left(\rho \frac{\partial}{\partial z} \frac{1}{\rho} \frac{\partial}{\partial z}+k^{2}-k_{0}^{2}\right) .
$$

This is the same eigenvalue equation that we obtain from the separation of variables solution of the Helmholtz equation, but this particular form is useful for deriving PE approximations. Equation 4 can be rewritten in terms of the depthoperator, $X$, as follows:

$$
\begin{aligned}
& p_{\Sigma}(r, z)=\sqrt{\frac{2 \pi i}{r}} \sum_{m=1}^{N} A_{m} e^{-i k_{0} \Delta r_{m} \sqrt{1+X}} \\
& \times\left[k_{0}^{-1 / 2}(1+X)^{-1 / 4} e^{i k_{0} r \sqrt{1+X}} \delta\left(z-z_{0}\right)\right],
\end{aligned}
$$

where the completeness relation for the normal modes has been used to obtain the delta function $\sum \psi_{n}^{*}\left(z_{0}\right) \psi_{n}(z)=$ $\delta\left(z-z_{0}\right)$.

The term in brackets in Equation 7 is actually the PE self-starter field for a point-source, $p_{s}(r, z)$ [3, Eq. 7], with a cylindrical spreading term $\left(r^{-1 / 2}\right)$ factored out. If we denote the range-factored (i.e., multiplied by $r^{1 / 2}$ ) self-starter as

$$
\begin{aligned}
p_{s}^{\prime}(r, z)= & \sqrt{r} p_{s}(r, z) \\
= & \sqrt{\frac{2 \pi i}{k_{0}}}(1+X)^{-1 / 4} e^{i k_{0} r \sqrt{1+X}} \\
& \times \delta\left(z-z_{0}\right),
\end{aligned}
$$

then we obtain the following result for the PE starting field at range $r_{s}$ from an array:

$$
p_{\Sigma}\left(r_{s}, z\right)=\frac{1}{\sqrt{r_{s}}} \sum_{m=1}^{N} A_{m} p_{s}^{\prime}\left(r_{s}-\Delta r_{m}, z\right) .
$$


That is, the array starting field in Equation 9 is obtained by summing together the $N$ point-source, range-factored starting fields together at range $r_{s}$, each displaced by distance $\Delta r_{m}$ from the origin. The resulting starting field may then be propagated forward from range $r_{s}$ in the usual fashion.

Although Equation 9 was derived for the far-field case, there is no requirement that the starter range, $r_{s}$, be in the farfield of the array. This is because the starting field, $p_{\Sigma}\left(r_{s} ; z\right)$, exhibits the same far-field radiation pattern as the source array in the Fraunhofer zone. Thus, even if the starting field is computed in the near-field, the array starter solution will converge to the correct far-field solution as the PE is marched out in range.

In order to avoid having to perform back-propagation, the range of the starting field, $r_{s}$, must be greater than the maximum value of $\Delta r_{m}$. The starting range must also be sufficiently large so that the numerical computation of the self-starter is stable for the smallest value of $r-\Delta r_{m}$. In the authors' experience, a suitable value for $r_{s}$ may be obtained by adding at least half the horizontal computation grid spacing to the maximum value of $\Delta r_{m}$. Also, if the array spacing is larger than the computation grid spacing (e.g., at high frequencies), then it is acceptable to take multiple PE range steps to arrive at $r_{s}$. Finally, note that $r_{s}$ may be different for different frequencies and azimuth angles.

Even though it was derived for the range-independent case, the array starter can be applied equally well to a rangedependent environment. This is because the normal modes that contribute to the starting field are excited according to the vertical wavenumber spectrum of the source array (i.e., the vertical directionality), as can be seen by inspection of Equation 4. As a consequence, the array starter can be used to compute the field from an array in a range-dependent environment as long as the environment is at least approximately range-independent near the source (i.e., within range $r_{s}$ of the source).

\section{VALIDATION}

Provided the modeler has access to an existing PE self-starter code - such as the one in RAM - numerical implementation of the array starter (Equation 9) is straightforward. The array starter code need only invoke the self-starter as a subroutine to generate multiple vertical starting fields (i.e., $p\left(r_{s}, z\right)$ ) at $N$ ranges

$$
r_{s m}=r_{s}-\Delta r_{m}, \quad m=1 \ldots N
$$

The resulting collection of starting fields must then be range-factored and summed together with the appropriate frequency-domain complex amplitudes $A_{m}(f)$ to yield the array starting field. The array starter has been implemented here using the RAM split-step Padé PE code [4], version $1.5 \mathrm{~g}$.

In order to validate the present implementation of the array starter, a range-independent benchmark test scenario was created using the ORCA normal-mode code [5], version 2.0. In a range-independent environment, Equation 2 is an exact expression for the pressure field from an array source, which

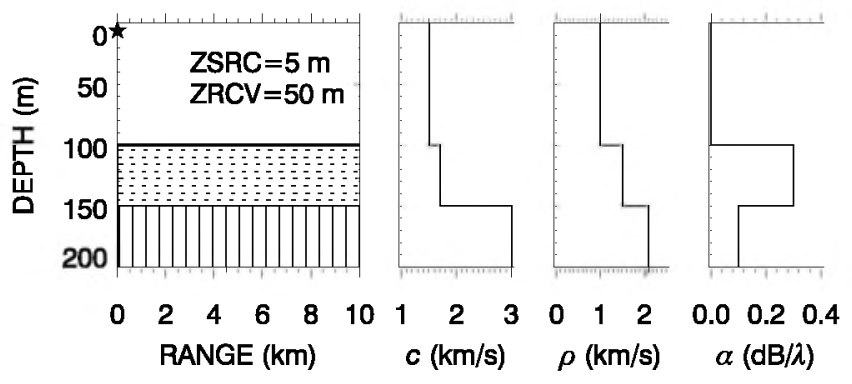

Figure 1: Diagram showing acoustic properties of the range-independent test case used for validating the array starter. Acoustic parameters are sound speed, $c$, density, $\rho$, and attenuation, $\alpha$. The source depth for the test case was $5 \mathrm{~m}$ and the receiver depth was $50 \mathrm{~m}$.

is valid even in the near-field (the array starter is only valid in the far-field). Thus benchmark reference solutions were generated for the array starter by using Equation 2 with the normal modes computed by ORCA.

The benchmark test case consisted of a planar array source in a shallow, range-independent ocean waveguide. The water depth in the waveguide was taken to be $100 \mathrm{~m}$ and the bottom consisted of a single 50 meter sediment layer over a semiinfinite basement. A diagram showing the acoustic properties of the test environment is presented in Figure 1. For simplicity, sound speed gradients were not used in each layer in order to avoid difficulties associated with the different sound speed interpolation methods used by RAM and ORCA (i.e., $c$-linear versus $1 / c^{2}$-linear interpolation).

The source, shown in Figure 2, was taken to be a 16 element planar array consisting of two identical sub-arrays separated by 10 metres. The sub-array elements were separated by $3 \mathrm{~m}$ and the tow depth of the array was taken to be $5 \mathrm{~m}$. This particular layout, with equally spaced elements divided into linear sub-arrays, was chosen because it is similar to commonly used airgun array configurations. The array elements all had identical amplitude and phase so that the main lobe of the array was in the vertical $\left(\phi= \pm 90^{\circ}\right)$ direction. Additionally, the source level of each element was taken to be $S L=-10 \log N \mathrm{~dB} @ 1 \mathrm{~m}(N=16)$ so that the vertical far-field source level of the array was $0 \mathrm{~dB} @ 1 \mathrm{~m}$ (unity amplitude). The propagation direction was taken to be $\theta=45^{\circ}$, as indicated by the arrow in Figure 2; this direction was purposely chosen to be at an angle with respect to the array axis so as to increase the complexity of the vertical directionality function and thus provide a more rigorous test case.

Figure 3 shows benchmark transmission loss comparisons for a receiver at $50 \mathrm{~m}$ depth. The transmission loss comparisons were run at two frequencies, $125 \mathrm{~Hz}$ and $500 \mathrm{~Hz}$, which correspond to quarter-wavelength and single-wavelength multiples of the sub-array element spacing. The plots show excellent agreement between the transmission loss computed using RAM and ORCA, indicating that the array starter is valid and that it was implemented correctly. 


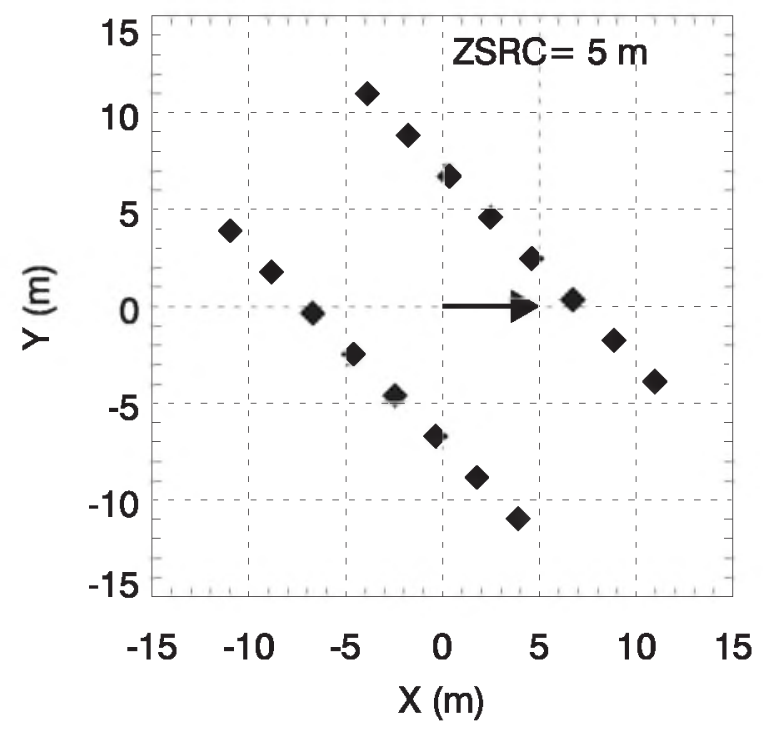

Figure 2: Diagram of the 16 element planar array used for the benchmark test scenario (the tow depth of the array is $\mathbf{5} \mathbf{~ m}$ ). The diamonds indicate the locations of the array elements and the arrow shows the direction of sound propagation (i.e., increasing $r$ ).

Figure 3 also shows the transmission loss for an isotropic point source with $S L=0 \mathrm{~dB} @ 1 \mathrm{~m}$, for comparison. Although the isotropic source has the same source level as the 16 element test array in the vertical direction $\left(\phi= \pm 90^{\circ}\right)$ the transmission loss for the point source is $\approx 12 \mathrm{~dB}$ less than for the array source in the horizontal direction. This example shows that, when modeling sound propagation from an array source, it is important to take directionality into account in order to to avoid substantial errors in the received sound level estimates.

\section{SYNTHETIC WAVEFORM EXAMPLE}

This section gives an example of a synthetic waveform calculation in a range-dependent test environment in order to demonstrate how the array starter method may be applied to a real airgun modeling problem. For this example calculation, a set of notional airgun signatures has been computed using a physical modeling approach. The source model employed here predicts notional airgun signatures by modeling the oscillation and radiation of a collection airgun bubbles and was developed by one of the authors as part of a thesis project [6]. In addition to the bubble oscillation physics, the source model includes non-linear pressure interaction between bubbles, port throttling and thermodynamic heat transfer across the bubble wall. The output of the airgun model has been validated against a large collection of source signature data for Bolt 600/B airguns [7]. The model physics are based on the work of investigators such as Ziolkowski [8,9], Laws et al. [10], and Landrø [11]. Note that, although modeled signatures have been used here, notional signatures for the array Canadian Acoustics / Acoustique canadienne starter method could also be obtained from near-field acoustic measurements of an airgun array. When using measured signatures, however, it is important to remove pressure interaction effects from near-field measurements to obtain the notional far-field signatures [12].

A typical 16-gun, two-string, $1500 \mathrm{in}^{3}$ (24.6 liter) airgun array was chosen for the example synthetic waveform calculation, as shown in Figure 4. The nominal tow depth of the example airgun array was taken to be $6 \mathrm{~m}$ and the firing pressure was taken to be 2000 psi $(13.6 \mathrm{MPa})$. Figure 5 shows the notional source signatures for this array as computed by the airgun source signature model. Distortion of the bubble pulses, particularly noticeable in the signatures of the $80 \mathrm{in}^{3}$ guns, is due to non-linear pressure interaction effects between airguns in the array [12]. Note that Figure 5 only presents source signatures for half of the guns in the array (i.e., a single string); the source signatures for the other eight guns are identical due to the symmetry of the array. The sampling interval of the synthetic source signature data presented in Figure 5 is $\Delta t=100 \mu \mathrm{s}$.

In order to perform waveform modeling using the Fourier synthesis method, a discrete frequency grid is employed

$$
f_{k}=k \Delta f, \quad k=1,2 \ldots M
$$

where $\Delta f$ is the frequency spacing of the field calculations and $f_{\text {max }}=M \Delta f$ is the maximum field computation frequency. Recall that the frequency spacing also determines the length of the synthetic data window according to the relation $T=1 / \Delta f$. For the example calculation presented here, $\Delta f=0.5 \mathrm{~Hz}$ and thus the length of the synthetic data window is $T=2 \mathrm{~s}$. The maximum computation frequency must be selected based on the power spectrum of the source waveforms; for the present example, $f_{\max }=1024 \mathrm{~Hz}$, which encompasses over $99.9 \%$ of the signal energy in the synthetic airgun waveforms shown in Figure 5.

In addition to the bandwidth of the field calculation, $f_{\max }$ also dictates the required frequency resolution for the source waveforms according to the relation $\Delta f=f_{\max } / M$. Thus, one must generally resample the airgun source signatures in the frequency domain so that their Discrete Fourier Transforms (DFT's) have the same frequency resolution as the field calculations. This is most simply accomplished by padding or truncating the source signature data in the time domain before taking the DFT. Once the source signatures have been resampled to the correct frequency spacing, the DFT coefficients correspond to the complex phase/amplitude terms, $A_{m}(k \Delta f)$, at each model frequency. Equation 9 may then be used to compute the starting field, at frequency $f$ along azimuth $\theta$, from the DFT coefficients and the projected airgun coordinates, $\Delta r_{m}(\theta)$. Consistent spatial sampling is also important: at each frequency, the range and depth spacing of the PE grid is taken to be an integer multiple of the smallest value, to ensure that the computation points are coincident.

Figure 6 shows the range-dependent test environment that has been used for the example waveform synthesis calculation. Acoustic propagation has been modeled along a downward sloping bottom, with water depth varying from $50 \mathrm{~m}$

Vol. 40 No. 1 (2012) - 22 

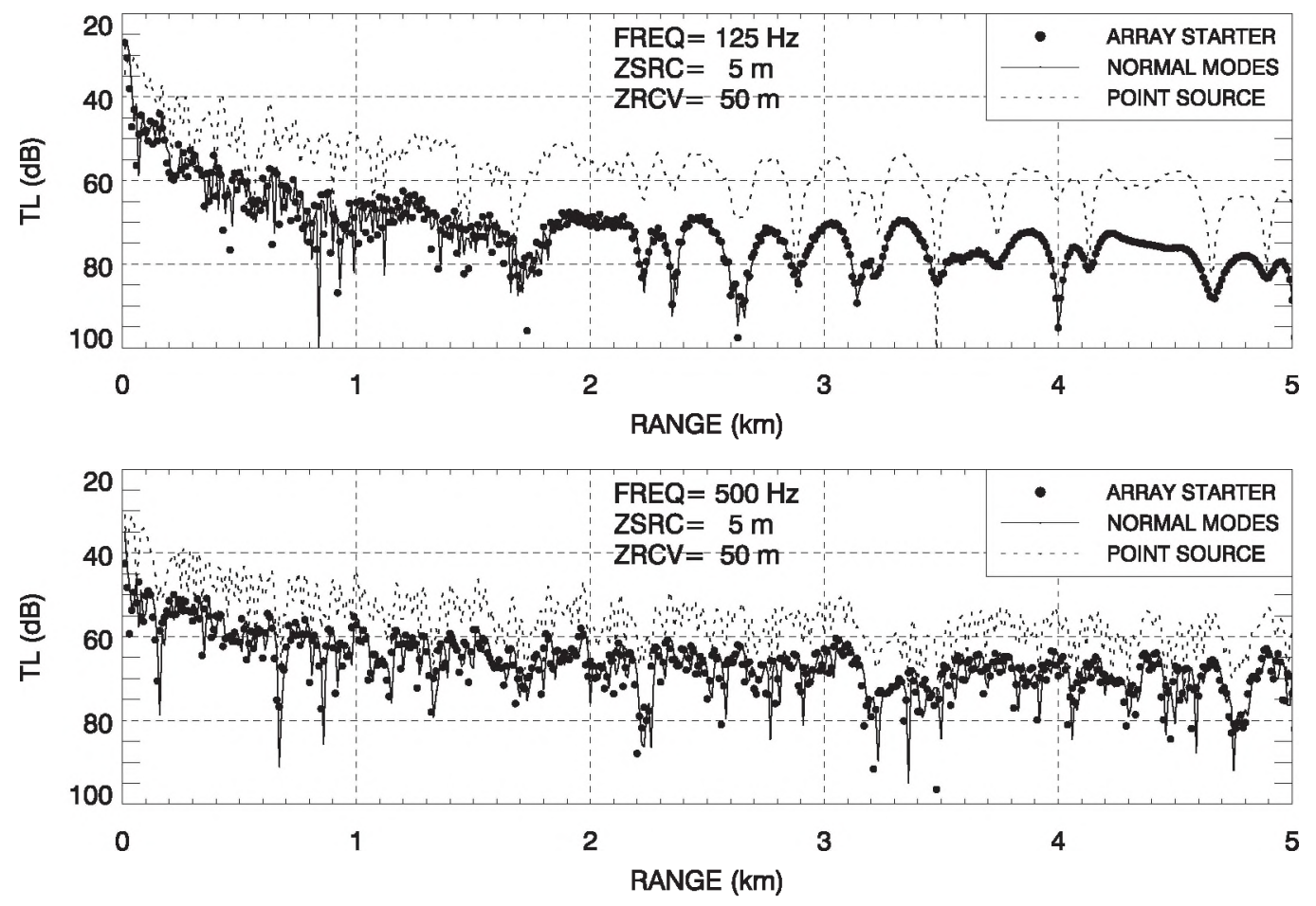

Figure 3: Benchmark comparison plots of transmission loss versus range for ORCA (solid line) and RAM seeded with the array starter (circles). Benchmark plots are presented for $125 \mathrm{~Hz}$ (top) and $500 \mathrm{~Hz}$ (bottom). For reference, the plots also show transmission loss for an isotropic point source.

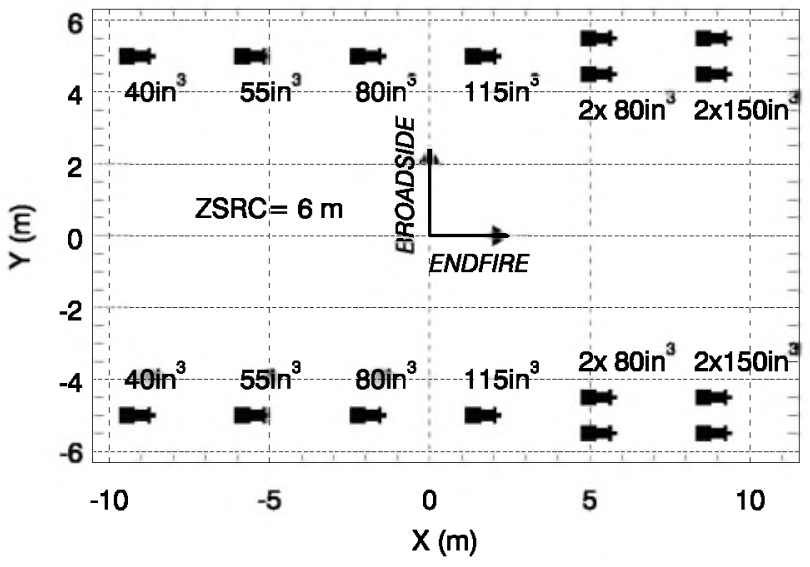

Figure 4: Plan-view diagram of the 16 airgun array used for the example calculation (total volume $1500 \mathrm{in}^{3}$ ); the nominal tow depth of the array was taken to be $6 \mathrm{~m}$. The plot annotations indicate the volume of each airgun and the arrows show the broadside and endfire directions from the array.
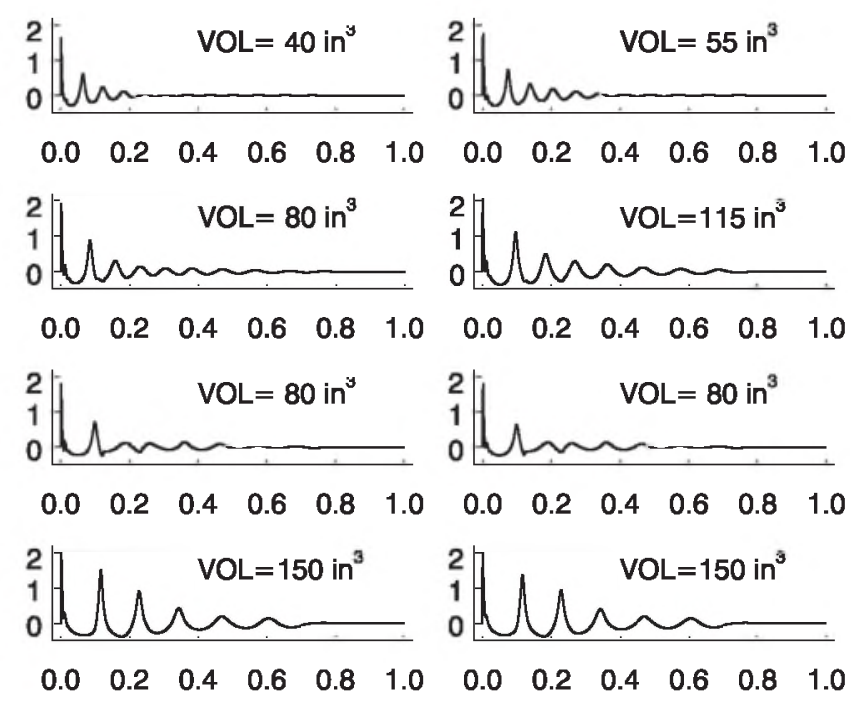

Figure 5: Notional source signatures for 8 of 16 guns in the 1500 in $^{3}$ example array, as computed by the source signature model. Pressure units (vertical axes) are in bar $\mathbf{m}$ $\left(1 \mathrm{bar}=10^{5} \mathrm{~Pa}\right.$ ) and time units (horizontal axes) are in seconds. The plot annotations indicate the volumes of individual guns. The source signatures for the remaining eight guns are identical to the ones presented here due to the symmetry of the array. 


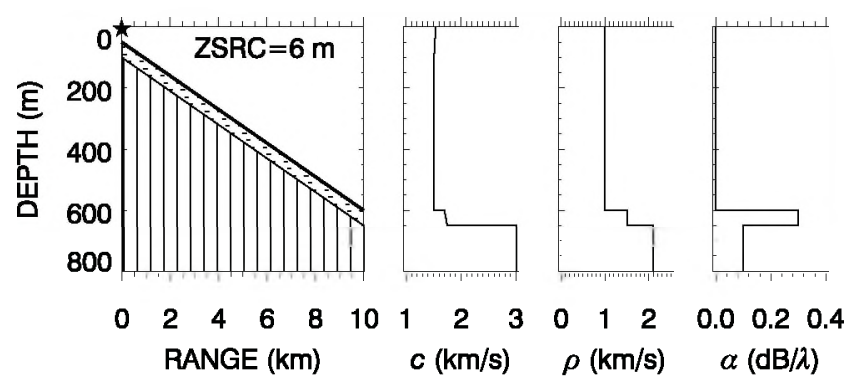

Figure 6: Diagram showing acoustic properties of the range-dependent environment used for the example waveform calculation. Acoustic parameters are sound speed, $c$, density, $\rho$, and attenuation, $\alpha$. Note that the sound speed profile in the water column is downward refracting.

at the source to $600 \mathrm{~m}$ at $10 \mathrm{~km}$ range $\left(3.15^{\circ}\right.$ slope $)$. The sound speed profile in the water column is downward refracting, varying from $1.54 \mathrm{~km} / \mathrm{s}$ at the sea-surface to $1.49 \mathrm{~km} / \mathrm{s}$ at $600 \mathrm{~m}$ depth. The sub-bottom, which runs parallel to the bathymetry, consists of a 50 meter sediment layer over a semiinfinite basement. The sound speed in the sediment layer is upward refracting with a vertical gradient of $1 / \mathrm{m}(c=1.70$ $1.75 \mathrm{~km} / \mathrm{s}$ ) and the sound speed in the basement is homogeneous $(c=3 \mathrm{~km} / \mathrm{s})$.

Figure 7 shows synthetic airgun pulse waveforms computed using the array starter method at $r=10 \mathrm{~km}$ for the range-dependent test environment of Figure 6 . Two cases are presented: one with array endfire $\left(\theta=0^{\circ}\right)$ oriented in the downslope direction and the other with array broadside $(\theta=$ $90^{\circ}$ ) oriented in the downslope direction. The plots show comparisons of airgun pulses at multiple receiver depths, from $50 \mathrm{~m}$ to $550 \mathrm{~m}$ in $100 \mathrm{~m}$ increments. Although the waveform data in Figure 7 are synthetic, they can be used to compute standard marine mammal noise exposure metrics, such as peak and rms sound pressure level, and sound exposure level (SEL), versus depth and range from the array, just as with acoustic data measured in situ.

The example waveforms presented in Figure 7 were computed assuming strictly two-dimensional sound propagation and also neglecting back-scattered energy from upslope of the array. Three-dimensional effects are unimportant for the example case presented here because the propagation plane is oriented directly downslope and so there is no horizontal coupling from adjacent azimuths [13]. The contribution of upslope back-scatter is expected to be negligible for the present example case, based on the work of Westwood [14] who showed that back-scattered energy was insignificant for penetrable wedge environments similar to the one considered here. Thus these two approximations are not expected to introduce significant errors into the waveform calcuations.

The synthesized waveforms in Figure 7 contain a considerable amount of structure due to the rich frequency content of the sound emissions from the airguns. From the figure, one can see that airgun pulses in the lower part of the waCanadian Acoustics / Acoustique canadienne ter column $(z>200 \mathrm{~m})$ have the greatest amplitudes and are much more sharply peaked than airgun pulses in the upper part of the water column $(z<200 \mathrm{~m})$. This is because high frequencies, which carry the energy from the sharp initial peaks in the airgun source signatures, are trapped in the bottom duct created by the down refracting sound speed profile. In the high frequency raytrace approximation, the sound rays "skip" down the seabed slope creating a shadow zone near the sea-surface. At low frequencies, on the other hand, the long wavelength normal modes span the whole water column, thus leaking low frequency sound energy out of the bottom duct. The low frequencies, however, only carry energy from the lower amplitude airgun bubble pulses, rather than the peaks.

Inspection of the waveforms in Figure 7 shows that levels in the broadside direction of the array are louder, and contain more high-frequencies, than levels in the endfire direction. This is because, for each of the two subarrays, all sound wavelengths add constructively in the broadside direction, whereas only those wavelengths that are integer multiples of the gun spacing (or are substantially larger than the gun spacing) add constructively in the endfire direction. This kind of directivity, with maximum levels measured at array broadside, is typical of seismic airgun arrays, which often consist of several gun strings towed in parallel behind the survey vessel. This example shows that the direction of sound propagation (i.e., the azimuth angle, $\theta$ ) is an important determiner of both the intensity and frequency content of the received pulse from an airgun array.

\section{CONCLUSION}

This article has presented an "array starter" technique for modeling sound propagation from an airgun array using the parabolic equation method. The array starter fully accounts for the vertical and horizontal directionality of an array source; it is computed by summing together phase-shifted, rangefactored replicas of the PE self-starter for each array element. Field predictions computed using the array starter are valid in the far-field of the array, including those regions where the acoustic field is dominated by steep propagation angles. A numerical implementation of the array starter was validated against exact (range-independent) field solutions for an array source computed using the ORCA normal mode model. An example was also presented of how the array starter may be combined with the Fourier synthesis technique to generate synthetic airgun waveform data in a range-dependent environment. Synthetic airgun pulses, computed using the array starter technique, can be used for predicting common noise exposure metrics for marine mammals, including peak and rms sound pressure level, and sound exposure level. Although the array starter was devised with airgun arrays in mind, this technique can be used for modeling sound propagation for any kind of horizontal or volumetric array source using the parabolic equation method.

Vol. 40 No. 1 (2012) - 24 

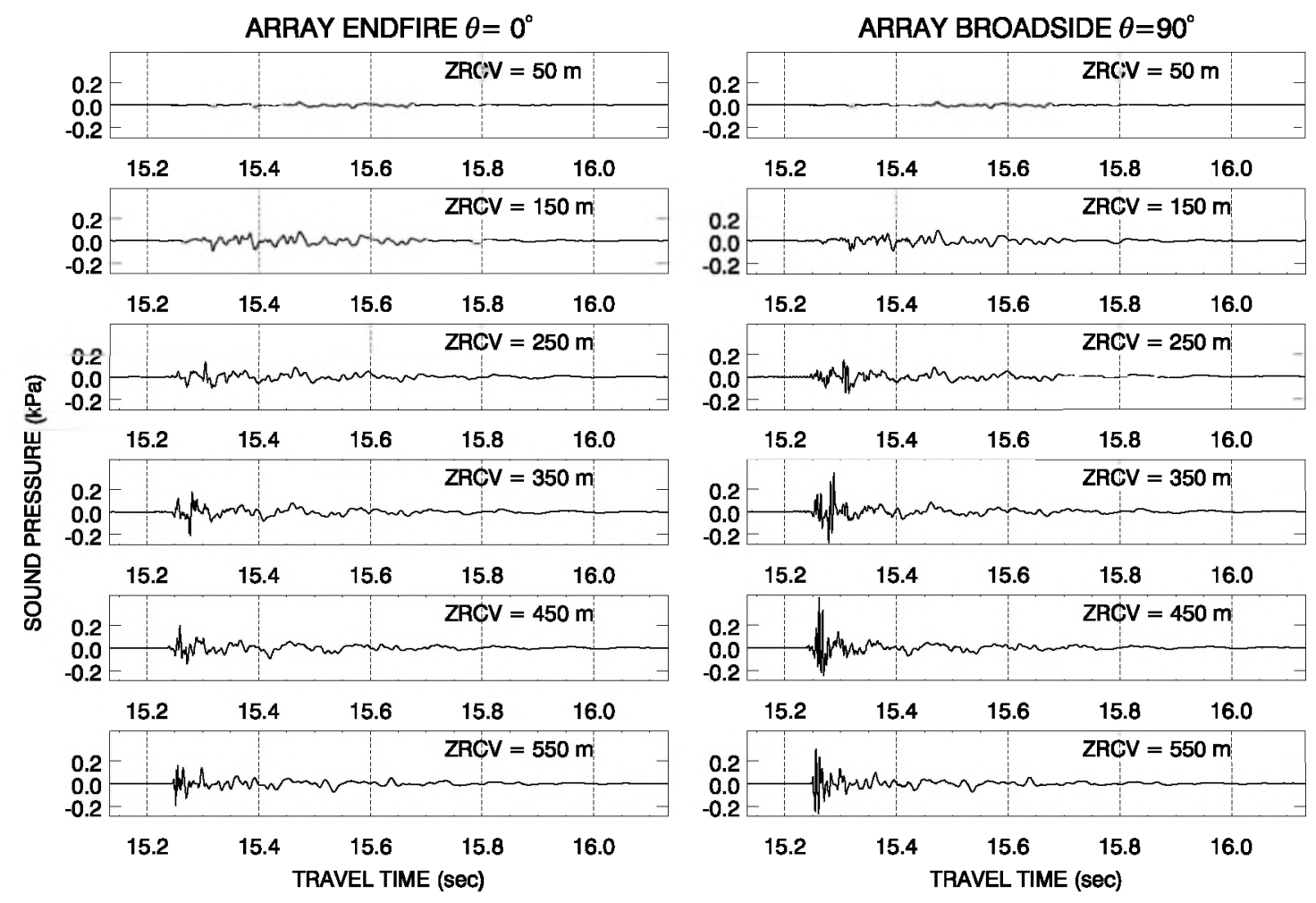

Figure 7: Synthetic airgun pulses at $10 \mathrm{~km}$ range, as computed in the range-dependent test environment of Figure 6, (left) for array endfire oriented downslope and (right) for array broadside oriented downslope. The receiver depth of each waveform is shown in the plot annotation. All waveforms are presented using the same time and pressure scales.

\section{REFERENCES}

[1] S. L. DeRuiter, P. L. Tyack, Y. T. Lin, A. E. Newhall, J. F. Lynch, P. J. O. Miller, "Modeling acoustic propagation of airgun array pulses recorded on tagged sperm whales (Physeter macrocephalus)" J. Acoust. Soc. Am. 120 4100-4114 (2006).

[2] H. Schmidt and F. B. Jensen, "A full wave solution for propagation in multilayered viscoelastic media with application to Gaussian beam reflection at fluid-solid interfaces" J. Acoust. Soc. Am. 77 813-825 (1985).

[3] M. D. Collins, "The stabilized self-starter" J. Acoust. Soc. Am. 106 1724-1726 (1999).

[4] M. D. Collins, "A split-step solution for the parabolic equation method" J. Acoust. Soc. Am. 94 1736-1742 (1993).

[5] E. K. Westwood, N. R. Chapman, and C. T. Tindle, "A normal-mode model for acousto-elastic ocean environments" J. Acoust. Soc. Am. 100 3631-3645 (1996).

[6] A. O. MacGillivray, "An acoustic modelling study of seismic airgun noise in Queen Charlotte Basin" M. Sc. Thesis, University of Victoria (2006).

[7] R. G. Racca and J. A. Scrimger, "Underwater acoustic source characterization of air and water guns" DREP Technical report No. 06SB 97708-5-7055, Defense Research and Development Canada (1986).

[8] A. Ziolkowski, "A method for calculating the output pressure waveform from an air gun" Geophys. J. Int. 21 137-161 (1970).
[9] A. Ziolkowski and G. Metselaar, "The pressure wave field of an air gun array" SEG Expanded Abstracts 3 274-276 (1984).

[10] M. Laws, L. Hatton, and M. Haartsen, "Computer modelling of clustered airguns" First Break 8 331-338 (1990).

[11] M. Landrø, "Modelling of GI gun signatures" Geophysical Prospecting 40 721-747 (1992).

[12] A. Ziolkowski, G. Parkes, L. Hatton, and T. Haugland, "The signature of an air gun array: computation from near-field measurements including interactions" Geophysics $471413-$ 1421 (1982).

[13] F. Sturm and J. A. Fawcett, "On the use of higher-order azimuthal schemes in 3-D PE modeling" J. Acoust. Soc. Am. 113 3134-3145 (2003).

[14] E. K. Westwood, "Ray model solutions to the benchmark wedge problems" J. Acoust. Soc. Am. 87 1539-1545 (1990). 


\section{Damaged hearing costs you dearly Preventing it doesn't}

Type 4448 - Helping to improve workplace noise assessment

\section{Simple reliability}

No cables, no connectors

\section{Forget it is there}

Secure shoulder mount with pin or clip attachment

\section{Ready when you are}

Long 28 hour battery-life

\section{Verify your Standards compliance}

HML option - verify hearing protection requirements

Works with Protector PC software - for intuitive analysis and reporting

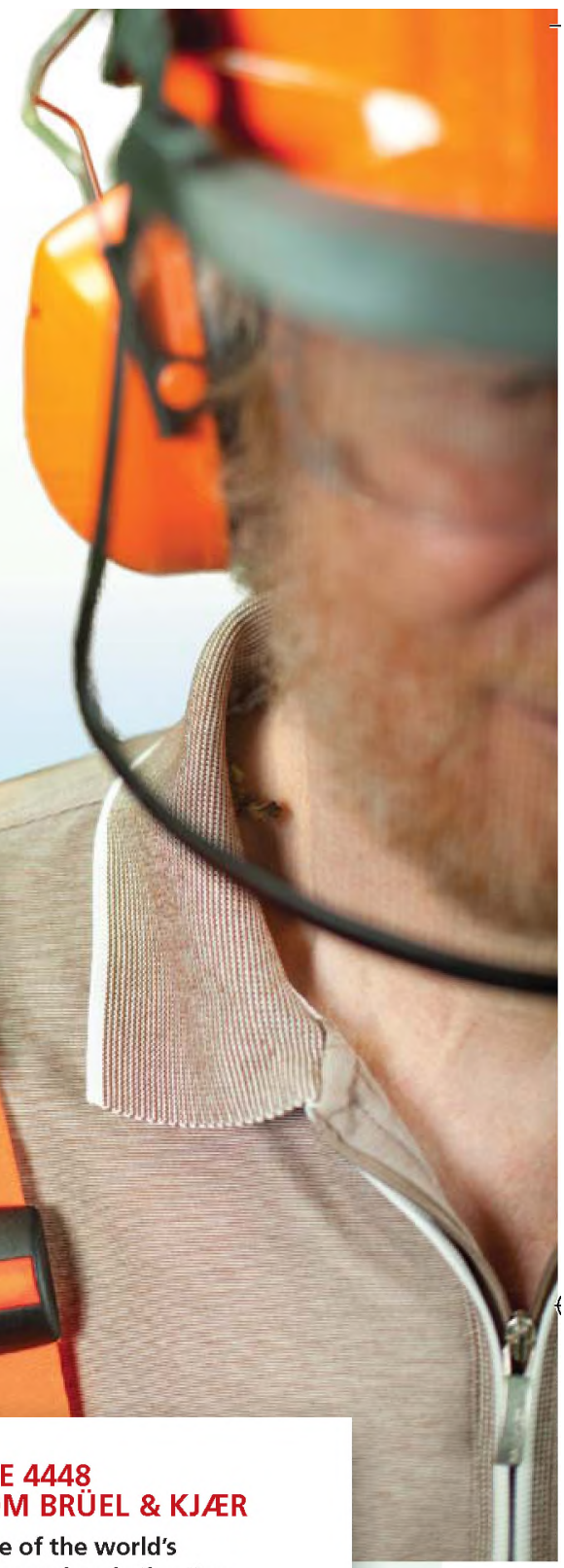

\section{FROM BRÜEL \& KJAER}

Home of the world's best sound and vibration instrumentation

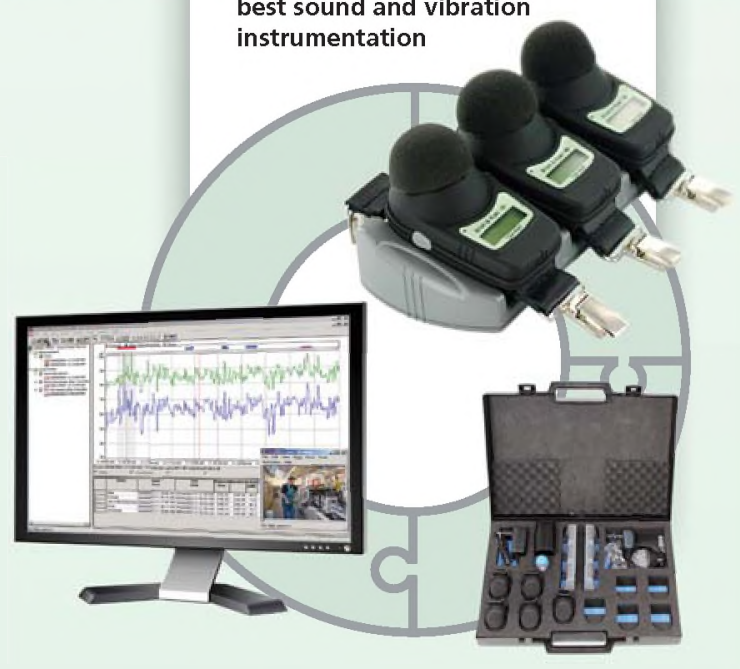

www.bksv.com/Type4448 